\title{
Interleukin-17-based cytokine signaling as a determinant for tumor immune microenvironment with prognostic value in triple-negative breast cancer
}

\author{
Victor C. Kok ( $\nabla$ vkok@alumni.harvard.edu ) \\ Kuang Tien General Hospital https://orcid.org/0000-0003-3440-8154 \\ Charles C.N. Wang \\ Asia University \\ Szu-Han Liao \\ Asia University \\ De-Lun Chen \\ Asia University
}

\section{Research Article}

Keywords: triple-negative breast cancer, immune cells, tumor microenvironment, IL-17 signaling pathway, immune evasion.

Posted Date: June 2nd, 2021

DOl: https://doi.org/10.21203/rs.3.rs-495771/v1

License: () (i) This work is licensed under a Creative Commons Attribution 4.0 International License. Read Full License

Version of Record: A version of this preprint was published at Breast Cancer: Targets and Therapy on April 12th, 2022. See the published version at https://doi.org/10.2147/BCTT.S359346. 


\section{Abstract}

Background: Only a proportion of triple-negative breast cancer (TNBC) is immunotherapy-responsive. We hypothesized that the tumor microenvironment (TME) might influence the outcomes of TNBC and investigated the relevant signaling pathways.

Methods: RNA-seq data of 115 TNBC samples and 112 normal adjacent tissues were retrieved for ESTIMATE, CIBERSORTX, X2K, KEGG, and GSVA analyses. The immune score (IS) and stromal score (SS) were calculated and correlated with the overall survival (OS) in TNBC. Finally, we validated the altered transcription factor (TF) genes in the cBioPortal.

Results: The SS is a good predictor of the OS (better survival in SS-low patients; $\mathrm{P}=0.0081$ ). In line with these results, when compared with IS-low/SS-high patients, IS-high/SS-low patients showed a better OS $(P=0.045)$. Moreover, macrophages were polarized toward the M2 phenotypes in IS-low/SS-high patients $(P<0.001)$. Compared with IS-low/SS-high patients, CIBERSORTx also showed that IS-high/SS-low patients had an increased number of memory B cells, CD8+ T cells ( $14.8 \%$ vs. $3.7 \%, p=0.0286)$, activated CD4+ memory T cells, follicular helper T cells, and activated NK cells in the TME; additionally, fewer resting NK cells were detected in the TME $(P=0.0108)$. Additionally, there were 284 upregulated and 367 downregulated DEGs (Differentially Expressed Genes) in IS-high/SS-low, and 187 upregulated and 183 downregulated DEGs in IS-low/SS-high patients. KEGG analysis further revealed that the DEGs were enriched in the IL-17 and cytokine-cytokine receptor interaction pathways. Of note, as per the cBioPortal platform, we discovered that $13 \%$ of ER-negative, HER2-unamplified BC patients harbored IL17RA deep deletions and 25\% harbored TRAF3IP2 amplifications; interestingly, the nine altered TF genes were associated with significantly worse relapse-free survival and OS, in the context of 2,377 and 4,819 BC patients, respectively.

Conclusions: The TME with different immune cell components influences the survival of TNBC patients. IS-high/SS-low patients show a better overall survival. Further studies are required to examine whether an immune/stromal state also predicts the response to immunotherapy.

\section{Introduction}

In 2020, the global incidence of breast cancer was 2,261,419 diagnosed across 185 countries, accounting for $11.7 \%$ of all cancer types [1]. Triple-negative breast cancer (TNBC) is characterized as estrogen receptor-negative, progesterone receptor-negative, and a lack of HER2 expression or amplification and accounts for approximately $15 \%-20 \%$ of all breast cancers [2,3]. TNBCs are heterogeneous (in terms of genomics, transcriptomics, and histopathology) and demonstrate the heterogeneity of response to antiprogrammed death-1/ligand-1 (anti-PD-1/PD-L1) checkpoint inhibition immunotherapy [3-5].

In fact, recent studies by Schmid et al. revealed that PD-1 or PD-L1 checkpoint blockade immunotherapy combined with neoadjuvant chemotherapy (NAC) improved the pathological complete response (pCR) rate in the neoadjuvant setting; therefore, they proposed such an approach would probably improve the 
progression-free survival (PFS) of patients with advanced or metastatic TNBC staining positive for PD-L1 in tumor-infiltrating immune cells, if used as the first-line therapy [6-8]. In contrast, other tumors are weakly immunogenic depending on the composition of the infiltrating immune cell populations and extrinsic factors (e.g. different metabolites or specific cytokines) enriched in the immune tumor microenvironment (TME). Of note, recent bioinformatics research, based on bulk tumor gene expression data demonstrated that a low abundance of regulatory CD4+ T cells (Treg) was significantly associated with an increased pCR rate in TNBC patients after NAC [9].

In a TNBC surgical specimen, untreated tumor cells typically represent approximately $60 \%$ of the cellular component, lymphoid and immune cells account for $20 \%$, and stromal cells such as fibroblasts, histiocytes, endothelial cells, myofibroblasts, and adipocytes represent the remaining $20 \%$ [10]. The primary function of stromal cells is to establish an immune response. The TME creates a chemokine-rich milieu inside, promoting the encounter between the boundary tumor cells and a variety of surrounding infiltrating immune cells in addition to cancer-associated fibroblasts, neo-vessels, neo-neurites, and other supportive tissues [11]. Prior research has demonstrated that the prognosis of TNBC in terms of diseasefree survival and disease-specific survival is worse in the basal-like immunosuppressed subtype and fare better in the basal-like immune-activated subtype, indicating that the immune TME plays a crucial role in the formation of either a tumor-permissive or tumor-expulsive milieu [4]. A clinicopathological study also demonstrated that TNBC with a high number of tumor-infiltrating CD56-positive natural killer (NK) cells was associated with a more favorable disease-free survival [12]. Additionally, research focusing on cancer-associated chemokines revealed that NK cells expressing abundant CXCR3 (also known as GPR9 and CD183) molecules on the cell surface are recruited by the chemokines CXCL9, CXCL10, or CXCL11 secreted by immune cells or stromal cells in the TME [13]. However, it is still unclear what driving force explains the immunogenic or immunosuppressive phenotype in the TME in patients with TNBC. Hence, here, we aimed to investigate which infiltrating immune cell populations would be associated with the immune phenotype, and which signaling pathways could determine a subgroup of TNBC that is strongly immunogenic, thus, having a better prognosis. Lastly, we also aimed to determine whether transcription factor signatures derived from the RNA-seq data have prognostic value.

\section{Methods}

\section{Ethics statement and study design}

This human data-based research study leveraged multiple publicly accessible RNA-seq datasets containing only mature, anonymous, and de-identified genetic and demographic data. The Institute Review Board of Kuang Tien General Hospital approved the study with a Certificate of Approval numbered KTGH-10458. This study was performed in accordance with the Declaration of Helsinki.

The schematic diagram (Fig. 1) shows the study design and methodology adopted in this study. First, we downloaded from TCGA, a breast cancer gene expression RNA-seq dataset (TCGA-BRCA). Using this 
dataset, we sorted 115 patients with TNBC, characterized by the lack of estrogen receptors, progesterone receptors, and HER2 non-overexpression or HER2-FISH un-amplification.

\section{Estimation of the immune/stromal scores}

As mentioned above, the gene expression data and corresponding clinical information from a total of 1222 cases were retrieved from a publically available dataset (TCGA-BRCA) [14]. After extracting the ER, $\mathrm{PgR}$, and HER2 information of each sample, a total of 115 TNBC cases, 112 normal cases, and 994 nonTNBC cases were identified. To predict TNBC purity, we applied the Estimation of STromal and Immune cells in MAlignant Tumor tissues using Expression data (ESTIMATE) algorithm to the normalized expression matrix to determine the immune/stromal scores of each TNBC patients. The immune score (IS; derived from the immune signature of 141 genes) and stromal score (SS; derived from the stromal signature of 141 genes) were calculated using the r-ESTIMATE package in R [15]. This algorithm ranknormalizes and rank-orders a set of gene expression values in a given sample and calculates the empirical cumulative distribution function from the signature gene set of the remaining genes; therefore, ESTIMATE can calculate the SS and IS from the RNA-seq data. The algorithm also allows the combination of these scores into an estimate score, used to infer tumor purity, as per the following formula: tumor purity $=\cos (0.6049872018+0.0001467884 \times$ estimate score $)$ [15]

\section{Correlation analyses}

The overall survival (primary prognosis endpoint-based) was estimated using Kaplan-Meier survival analysis. Based on the calculated SS and IS, the corresponding patients were classified into two groups, and their prognoses were individually examined. A previously published method (maximally selected rank statistics), was employed for optimal cutoff identification using the survival in R to explore the relationship between the overall survival and the two groups of TNBC cases.

\section{CIBERSORT analysis}

We used CIBERSORT X (https://cibersort.stanford.edu) to compare the proportion of infiltrating immune cells in the TME of IS-low/SS-high and IS-high/SS-low TNBC patients $[16,17]$. The CIBERSORT accurately allows the evaluation of the relative levels of 22 immune cell phenotypes; such and analysis was performed using the immunedeconv package in $\mathrm{R}$ [18]. The immune cell fraction level divided by the cutoff value was 0 or 1 in the subsequent scoring formula.

\section{Differential expression analysis}

According to the ESTIMATE results, the intersection genes were selected based on the stromal/immune scores. The limma package in R was used to screen DEGs between normal and TNBC samples [19]. Genes with a $p$-value $\leq 0.0001$ and absolute log 2 fold-change $\geq 4$ were considered to be differentially expressed and extracted further for network construction. Heatmaps were generated using the pheatmap package in $\mathrm{R}$ [20]. The generated heatmaps and volcano plots show the differentially expressed genes in either the IS-high/SS-low or IS-low/SS-high TNBC groups. 


\section{Gene Ontology and KEGG Pathway Enrichment Analyses}

Using these DEGs defined as above for the two TNBC groups, we investigated the enriched signaling pathways based on GO terms and KEGG pathways. Functional enrichment analyses of GO terms, including cellular components, molecular functions, and biological processes, and KEGG pathways were performed using the clusterProfiler package in $\mathrm{R}$ [21]. The functions among genes of interest were adjusted with a cutoff criterion of $p<0.05$.

\section{eXpression2Kinases analysis}

We utilized eXpression2Kinases (X2K), as reported elsewhere, to disclose the potential enrichment of transcription factors $[22,23]$.

\section{Gene set variation analysis}

Gene set variation analysis (GSVA) is a bioinformatics framework that organizes gene expression data in the form of a pathway or signature summary [24]. GSVA is a popular pathway-related immune infiltration and tumor mutational burden immune-related analysis. Here, we leveraged GSVA to provide an accurate definition of pathway enrichment between samples from different groups. We used the GSVA package in $R$ to evaluate the $t$ score and assigned the pathway activity conditions. We then used the pheatmap package in R to display the distinctions in pathway activation between normal tissues and those of IShigh/SS-low and IS-low/SS-high patients.

\section{cBioPortal for cancer genomics}

Finally, we validated our findings in a different, more extensive dataset of breast cancer via cBioPortal analysis $[25,26]$.

\section{Results}

From the TCGA Breast Cancer dataset, gene expression data for 115 patients with triple-negative breast cancer and 112 normal adjacent tissues were retrieved. Fig. 1 demonstrates the study design, flow, and methodology used in the current study. In this cohort of 115 TNBC patients, favorable overall survival as per the Kaplan-Meier curves was significantly correlated with an earlier disease stage $(P=0.0012$; Fig. 2$)$.

The ESTIMATE algorithm computationally estimates the fraction of stromal and immune cells in the TME. The SS was high (SS-high) in 10 patients, and the IS was high (IS-high) in the ten other patients. There were no significant correlations between the SS or IS and the cancer stage (Suppl. Fig. 1). However, as per the Kaplan-Meier analyses, SS-low patients showed a higher overall survival (OS) than that of SShigh patients ( $P=0.0081$; Suppl. Fig. 2), while IS-high patients showed a higher OS than that of IS-low patients $(P=0.2$; too few cases in the IS-high; Suppl. Fig. 3). Of note, a strong correlation between both the SS and IS and the patients' overall prognosis was observed. Expectedly, when compared with ISlow/SS-high patients, IS-high/SS-low patients showed a better OS $(P=0.045)($ Fig. 3). Cytoscape 
revealed the immune cell infiltration levels between samples grouped by IS-high/SS-low or IS-low/SS-high (Suppl. Fig. 4). Interestingly, grouped linear regression showed a statistically significant increase in M2 macrophages in TNBC patients with the IS-high/SS-low phenotype. In line with these results, in the tumor microenvironment (TME), a higher proportion of M2 was also found in IS-low/SS-high patients, as estimated by CYBERSORTx ( $<$ < 0.001) (Suppl. Fig. 5). Additionally, significantly more CD8+ cytotoxic Tcells, memory $B$ cells $(P=0.0304)$, activated $C D 4+$ memory T cells $(P=0.0056)$, follicular helper $T$ cells $(P$ $=0.0044)$, and activated NK cells $(P=0.0511)$ were also observed in IS-high/SS-low patients $(14 \%$ vs. $5.3 \%, p=0.0143$ ) (Table 1$)$. On the other hand, more resting NK cells were observed in IS-low/SS-high patients $(P=0.0108)$.

Moreover, DEG analysis showed 651 DEGs (284 upregulated, and 367 downregulated) in IS-high/SS-low patients, and 370 DEGs (187 upregulated, and 183 downregulated) in IS-low/SS-high patients (Suppl. Fig. 6). Heatmaps and volcano plots of the DEGs in the context of these two groups are shown in Fig. 4A-D. KEGG pathway enrichment analysis showed that the overexpressed DEGs from both phenotypes were enriched in the IL-17 signaling pathway and viral protein interaction cytokine and cytokine receptor genes. Notably, the overexpressed DEGs in the context of the SS-low/IS-high phenotype were also enriched in other two cytokine-related pathways: cytokine-cytokine receptor interactions pathway and chemokine signaling pathway (Fig. 4E).

Using $\mathrm{X} 2 \mathrm{~K}$, we also inferred the transcription factors associated with the two immune phenotypes. In SShigh/IS-low TNBC patients, the inferred TFs were PPARG, HNF4A (also known as farnesoid X receptor, FXR), NR1H4, NROB2, and MLXIPL, whereas the TF signature of SS-low/IS-high TNBC patients was composed of the PPARG, CEBPA, and MLXIPL TFs (Fig. 5). Of note, the OncoScore analysis did not perform as well in the search for the TF signatures (Suppl. Table 1). Additionally, we performed a manual search on PubMed and discovered nine TF genes linked to IL-17-mediated signaling, including PPARG, CEBPA, MEOX1, KLF15, CD36, ZNF750, EZH2, HNF4A, and NR0B2 (Table 2).

Importantly, GSVA suggested the involvement of additional pathways, including the JAK-STAT signaling, T cell receptor signaling, B cell receptor signaling, cytokine-cytokine receptor interaction, TGF- $\beta$ signaling, and PPAR signaling pathways (Fig. 6; the upper panel shows the GSVA of the 370 DEGs of the SShigh/IS-low subgroup and the lower panel shows the GSVA of the 651 DEGs of the SS-low/IS-high subgroup).

\section{Validation of the results in the cBioPortal}

IL-17 is a proinflammatory cytokine that signals mainly via the TRAF3 Interacting protein 2 (TRAF3IP2), as reported previously $[27,28]$. TRAF3IP2 is an inflammatory mediator and upstream regulator of several crucial transcription factors, such as AP-1 and NF-KB [28]. Act1, an essential component in IL-17 signaling complex, is encoded by the gene TRAF3IP2. Finally, we used the Oncoprint from cBioPortal to validate the frequency of IL-17 genes in TNBC. We discovered that 13\% of ER-negative and HER2-FISH unamplified breast cancers harbored IL17RA deep deletions and 25\% harbored TRAF3IP2 amplifications (Suppl. Fig 7). 
Interestingly, we also discovered using the cBioPortal platform that aberrations in the nine TF genes mentioned above are associated with a worse prognosis, as per the relapse-free survival of 2,377 patients (log-rank, $P=0.00007$ ) and the overall survival of a larger group of 4,819 patients (log-rank, $P=$ 0.001697) (Fig. 7).

\section{Discussion}

Several recent studies have shed light on the pivotal role of the TME in the shaping of the tumor behavior in TNBC [29-34]. Given the paucity of studies investigating the mechanism behind the strikingly different prognoses of immunosuppressive or immunogenic TNBCs, our study aimed to explore the differences in stromal cells (particularly immune cells) within the TME, as well as in the enriched DEGs in the search for the driving force behind the formation of these two different phenotypes. Interestingly, we discovered that the composition of immune cells reacting to TNBC tumor cells was strikingly different in the highly immunogenic-weak stromal TME and the strong stromal-weak immunogenic TME. Additionally, we discovered that the immunosuppressive type (SS-high/IS-low) showed gene signatures enriched in the IL17 , and viral-cytokine and cytokine receptor interactions' pathways. Of note, this study is unique considering the demonstration that altered TF genes derived from IL-17-mediated signaling showed strong significance with respect to both the relapse-free and overall survival, as per the analysis of another extensive dataset.

More than 1,600 known (or likely) human TF genes represent approximately $8 \%$ of the human genes [35]. Mutations in TF genes are often highly deleterious in humans [35]. Nine TF genes, PPARG, CEBPA, MEOX1, CD36, ZNF750, KLF15, EZH2, HNF4A, and NROB2, were enriched in our bioinformatics analysis. Importantly, this is the first time that the alteration in one or more of the nine TF in the context of TNBC was linked to the IL-17 signaling pathway and associated with significantly poorer prognosis in terms of relapse-free survival and overall survival in a large number of breast cancer patients, excluding ERpositive and HER2-amplified cases.

A plethora of studies have revealed increased IL-17A levels in ER-negative or triple-negative breast cancer [36]. In fact, the upregulation of IL-17A signaling is associated with increased expression of programmed cell death protein 1 (PD-1) and programmed death-ligand 1 (PD-L1) in breast cancer with low ER expression, which may elevate the infiltration of CD8+ T cells in the tumor tissues [36]. Of note, CD4+ T cells among tumor-infiltrating lymphocytes in the TME are the main source of IL-17 [37, 38]. IL-17mediated downstream signaling plays a critical role in the TME, inducing the expression of different genes either to switch on pro-tumor effector cytokines or to inhibit tumor growth in a context- and systemdependent manner [37]. For example, IL-17 induces the production of IL-6, which in turn induces STAT3 (signal transducer and activator of transcription 3) [37]; in fact, IL-6 orchestrates both the increase in the recruitment of suppressive tumor-associated myeloid cells and impacts their ability to inhibit anti-tumor T-cell responses [39]. Early on, a recent study uncovered how an IL-17-mediated paracrine network could recruit immature myeloid cells into the TME, causing tumor progression; this study demonstrated that through NF-KB and ERK signaling, tumor-infiltrating T helper type 17 (Th17) cells and IL-17 would induce 
granulocyte colony-stimulating factor expression, which is crucial for the mobilization of immature myeloid cells into the TME [30]. In contrast, Bianchini et al. demonstrated that a subset of highly proliferative, ER-negative breast cancers with high expression of a B-cell/plasma cell stromal metagene corresponding to immune functions and extracellular matrix components were associated with a favorable prognosis [10]. These studies lend support to the composition of cellular constituents in the $\mathrm{TME}$, and their association with the patient's prognosis.

Still regarding the TF genes associated with TNBC, a previous study suggested that EZH2 plays decisive roles in immune cells (e.g., T cells, NK cells, dendritic cells, and macrophages) in the tumor microenvironment [40]. Liu and colleagues demonstrated a novel function of PPARy in lymphocyte trafficking and the cross-talk between Th17 and B cells [41].

Additionally, investigators from the Institut Curie and INSERM found that a high Th17 metagene was associated with a good prognosis in T cell non-inflamed type TNBC, suggesting Th17 is a novel prognostic composite biomarker. Altogether, these studies clearly support the notion that integrating immune cells and tumor molecular diversity is an efficient strategy for the prognostic stratification of cancer patients [42].

The purpose of the current research was neither to develop a predictive molecular panel biomarker, nor to identify innate resistance versus sensitive phenotypes to checkpoint inhibitor immunotherapy; we aimed to investigate the factors behind the TME's immune-stromal state and to look for the systemic functions of the involved molecular signaling pathways. However, this study is not without limitations. One of them is the available low number of cases used to separate tumors into two extreme immune-stromal phenotypes.

\section{Conclusions}

Overall, our data suggest that the disclosure of the distinct molecular anatomy of the TME in patients with TNBC (not of the cancer cells per se) will assist in the determination of the ultimate tumor behavior. Of note, the results of this integrated in silico study can only be generalized to approximately $17 \%$ of patients with TNBC, in which infiltrating stromal cells and immune cells play a determinant prognostic role. Remarkably, our data suggest that the determining factor for the differences in the tumor immune microenvironment (immunogenic versus immunosuppressive) can be explained by IL-17 signaling and its paracrine network.

\section{Declarations}

\section{Ethics approval and consent to participate}

This human data research leveraged multiple publicly accessible RNA-seq datasets containing only mature, anonymous, and de-identified genetic and demographic data. The Institute Review Board of 
Kuang Tien General Hospital approved the study with a Certificate of Approval numbered KTGH-10458. This study was performed in accordance with the Declaration of Helsinki.

Consent for publication

Not applicable

\section{Availability of data and materials}

The datasets used and/or analyzed during the current study are available from the corresponding author upon reasonable request.

\section{Competing interests}

The authors declare that they have no competing interests.

\section{Funding}

This study was partly supported by a research fund from the Ministry of Science and Technology, Taiwan, with the grant number as MOST 109-2321-B-468-001. The funder had no role in study design, data collection and analysis, decision to publish, or preparation of the manuscript.

\section{Authors' contributions}

VCK conceived the study. VCK and CCW designed the study. SHL and DLC performed the experiments. All authors analyzed and interpreted the results, and VCK was the major contributor to the writing of the manuscript. All authors read and approved the final manuscript.

\section{Acknowledgements}

The results published here are based on data generated by the TCGA Research Network: https://www.cancer.gov/tcga.

\section{References}

1. Sung H, Ferlay J, Siegel RL, Laversanne M, Soerjomataram I, Jemal A, Bray F: Global cancer statistics 2020: GLOBOCAN estimates of incidence and mortality worldwide for 36 cancers in 185 countries. CA Cancer J Clin 2021.

2. Glodzik D, Bosch A, Hartman J, Aine M, Vallon-Christersson J, Reutersward C, Karlsson A, Mitra S, Nimeus $\mathrm{E}$, Holm $\mathrm{K}$ et al: Comprehensive molecular comparison of BRCA1 hypermethylated and BRCA1 mutated triple negative breast cancers. Nat Commun 2020, 11(1):3747.

3. Marra A, Trapani D, Viale G, Criscitiello C, Curigliano G: Practical classification of triple-negative breast cancer: intratumoral heterogeneity, mechanisms of drug resistance, and novel therapies. NPJ Breast Cancer 2020, 6:54. 
4. Burstein MD, Tsimelzon A, Poage GM, Covington KR, Contreras A, Fuqua SA, Savage MI, Osborne CK, Hilsenbeck SG, Chang JC et al: Comprehensive genomic analysis identifies novel subtypes and targets of triple-negative breast cancer. Clin Cancer Res 2015, 21(7):1688-1698.

5. Kok VC: Hepatic metastasectomy and paclitaxel provide long-term survival for a young woman with recurrent triple-negative metastatic breast cancer: 16 years follow-up. J Cancer Res Ther 2018, 14(3):722-723.

6. Schmid P, Adams S, Rugo HS, Schneeweiss A, Barrios CH, Iwata H, Diéras V, Hegg R, Im SA, Shaw Wright $\mathrm{G}$ et al: Atezolizumab and Nab-Paclitaxel in Advanced Triple-Negative Breast Cancer. N Engl J Med 2018, 379(22):2108-2121.

7. Schmid P, Cortes J, Pusztai L, McArthur H, Kümmel S, Bergh J, Denkert C, Park YH, Hui R, Harbeck N et al: Pembrolizumab for Early Triple-Negative Breast Cancer. N Engl J Med 2020, 382(9):810-821.

8. Schmid P, Rugo HS, Adams S, Schneeweiss A, Barrios CH, Iwata H, Diéras V, Henschel V, Molinero L, Chui SY et al: Atezolizumab plus nab-paclitaxel as first-line treatment for unresectable, locally advanced or metastatic triple-negative breast cancer (IMpassion130): updated efficacy results from a randomised, double-blind, placebo-controlled, phase 3 trial. Lancet Onco/ 2020, 21(1):44-59.

9. Oshi M, Asaoka M, Tokumaru Y, Angarita FA, Yan L, Matsuyama R, Zsiros E, Ishikawa T, Endo I, Takabe K: Abundance of Regulatory T Cell (Treg) as a Predictive Biomarker for Neoadjuvant Chemotherapy in Triple-Negative Breast Cancer. Cancers (Basel) 2020, 12(10).

10. Bianchini G, Qi Y, Alvarez RH, Iwamoto T, Coutant C, Ibrahim NK, Valero V, Cristofanilli M, Green MC, Radvanyi $\mathrm{L}$ et al: Molecular anatomy of breast cancer stroma and its prognostic value in estrogen receptor-positive and -negative cancers. J Clin Oncol 2010, 28(28):4316-4323.

11. Kok VC: Current Understanding of the Mechanisms Underlying Immune Evasion From PD-1/PD-L1 Immune Checkpoint Blockade in Head and Neck Cancer. Front Oncol 2020, 10:268.

12. Bouzidi L, Triki H, Charfi S, Kridis WB, Derbel M, Ayadi L, Sellami-Boudawara T, Cherif B: Prognostic Value of Natural Killer Cells Besides Tumor-Infiltrating Lymphocytes in Breast Cancer Tissues. Clin Breast Cancer 2021.

13. Nagarsheth $\mathrm{N}$, Wicha MS, Zou W: Chemokines in the cancer microenvironment and their relevance in cancer immunotherapy. Nat Rev Immunol 2017, 17(9):559-572.

14. Wang CCN, Li CY, Cai JH, Sheu PC, Tsai JJP, Wu MY, Li CJ, Hou MF: Identification of Prognostic Candidate Genes in Breast Cancer by Integrated Bioinformatic Analysis. J Clin Med 2019, 8(8).

15. Yoshihara K, Shahmoradgoli M, Martinez E, Vegesna R, Kim H, Torres-Garcia W, Trevino V, Shen H, Laird PW, Levine DA et al: Inferring tumour purity and stromal and immune cell admixture from expression data. Nat Commun 2013, 4:2612.

16. Newman AM, Liu CL, Green MR, Gentles AJ, Feng W, Xu Y, Hoang CD, Diehn M, Alizadeh AA: Robust enumeration of cell subsets from tissue expression profiles. Nat Methods 2015, 12(5):453-457.

17. Newman AM, Steen CB, Liu CL, Gentles AJ, Chaudhuri AA, Scherer F, Khodadoust MS, Esfahani MS, Luca BA, Steiner $D$ et al: Determining cell type abundance and expression from bulk tissues with digital cytometry. Nat Biotechnol 2019, 37(7):773-782. 
18. Sturm G, Finotello F, List M: Immunedeconv: An R Package for Unified Access to Computational Methods for Estimating Immune Cell Fractions from Bulk RNA-Sequencing Data. Methods Mol Biol 2020, 2120:223-232.

19. Ritchie ME, Phipson B, Wu D, Hu Y, Law CW, Shi W, Smyth GK: limma powers differential expression analyses for RNA-sequencing and microarray studies. Nucleic Acids Res 2015, 43(7):e47.

20. Galili T, O'Callaghan A, Sidi J, Sievert C: heatmaply: an R package for creating interactive cluster heatmaps for online publishing. Bioinformatics 2018, 34(9):1600-1602.

21. Yu G, Wang LG, Han Y, He QY: clusterProfiler: an R package for comparing biological themes among gene clusters. Omics 2012, 16(5):284-287.

22. Chen EY, Xu H, Gordonov S, Lim MP, Perkins MH, Ma'ayan A: Expression2Kinases: mRNA profiling linked to multiple upstream regulatory layers. Bioinformatics 2012, 28(1):105-111.

23. Clarke DJB, Kuleshov MV, Schilder BM, Torre D, Duffy ME, Keenan AB, Lachmann A, Feldmann AS, Gundersen GW, Silverstein MC et al: eXpression2Kinases (X2K) Web: linking expression signatures to upstream cell signaling networks. Nucleic Acids Res 2018, 46(W1):W171-w179.

24. Hänzelmann S, Castelo R, Guinney J: GSVA: gene set variation analysis for microarray and RNA-seq data. BMC Bioinformatics 2013, $14: 7$.

25. Cerami E, Gao J, Dogrusoz U, Gross BE, Sumer SO, Aksoy BA, Jacobsen A, Byrne CJ, Heuer ML, Larsson $\mathrm{E}$ et al: The cBio cancer genomics portal: an open platform for exploring multidimensional cancer genomics data. Cancer Discov 2012, 2(5):401-404.

26. Gao J, Aksoy BA, Dogrusoz U, Dresdner G, Gross B, Sumer SO, Sun Y, Jacobsen A, Sinha R, Larsson E et al: Integrative analysis of complex cancer genomics and clinical profiles using the cBioPortal. Sci Signal 2013, 6(269):pl1.

27. Alt EU, Wörner PM, Pfnür A, Ochoa JE, Schächtele DJ, Barabadi Z, Lang LM, Srivastav S, Burow ME, Chandrasekar B et al: Targeting TRAF3IP2, Compared to Rab27, is More Effective in Suppressing the Development and Metastasis of Breast Cancer. Sci Rep 2020, 10(1):8834.

28. Herjan T, Hong L, Bubenik J, Bulek K, Qian W, Liu C, Li X, Chen X, Yang H, Ouyang S et al: IL-17receptor-associated adaptor Act1 directly stabilizes mRNAs to mediate IL-17 inflammatory signaling. Nat Immunol 2018, 19(4):354-365.

29. Bareche Y, Buisseret L, Gruosso T, Girard E, Venet D, Dupont F, Desmedt C, Larsimont D, Park M, Rothé F et al: Unraveling Triple-Negative Breast Cancer Tumor Microenvironment Heterogeneity: Towards an Optimized Treatment Approach. J Natl Cancer Inst 2020, 112(7):708-719.

30. Chung AS, Wu X, Zhuang G, Ngu H, Kasman I, Zhang J, Vernes JM, Jiang Z, Meng YG, Peale FV et al: An interleukin-17-mediated paracrine network promotes tumor resistance to anti-angiogenic therapy. Nat Med 2013, 19(9):1114-1123.

31. Deng J, Thennavan A, Shah S, Bagdatlioglu E, Klar N, Heguy A, Marier C, Meyn P, Zhang Y, Labbe K et al: Serial single-cell profiling analysis of metastatic TNBC during Nab-paclitaxel and pembrolizumab treatment. Breast Cancer Res Treat 2020. 
32. Deng L, Lu D, Bai Y, Wang Y, Bu H, Zheng H: Immune Profiles of Tumor Microenvironment and Clinical Prognosis among Women with Triple-Negative Breast Cancer. Cancer Epidemiol Biomarkers Prev 2019, 28(12):1977-1985.

33. Vangangelt KMH, van Pelt GW, Engels CC, Putter H, Liefers GJ, Smit V, Tollenaar R, Kuppen PJK, Mesker WE: Prognostic value of tumor-stroma ratio combined with the immune status of tumors in invasive breast carcinoma. Breast Cancer Res Treat 2018, 168(3):601-612.

34. Zheng S, Zou Y, Xie X, Liang JY, Yang A, Yu K, Wang J, Tang H, Xie X: Development and validation of a stromal immune phenotype classifier for predicting immune activity and prognosis in triplenegative breast cancer. Int J Cancer 2020, 147(2):542-553.

35. Lambert SA, Jolma A, Campitelli LF, Das PK, Yin Y, Albu M, Chen X, Taipale J, Hughes TR, Weirauch MT: The Human Transcription Factors. Cell 2018, 172(4):650-665.

36. Shuai C, Yang X, Pan H, Han W: Estrogen Receptor Downregulates Expression of PD-1/PD-L1 and Infiltration of CD8(+) T Cells by Inhibiting IL-17 Signaling Transduction in Breast Cancer. Front Oncol 2020, 10:582863.

37. Wang L, Yi T, Kortylewski M, Pardoll DM, Zeng D, Yu H: IL-17 can promote tumor growth through an IL-6-Stat3 signaling pathway. J Exp Med 2009, 206(7):1457-1464.

38. Su X, Ye J, Hsueh EC, Zhang Y, Hoft DF, Peng G: Tumor microenvironments direct the recruitment and expansion of human Th17 cells. J Immunol 2010, 184(3):1630-1641.

39. Ruhland MK, Loza AJ, Capietto AH, Luo X, Knolhoff BL, Flanagan KC, Belt BA, Alspach E, Leahy K, Luo J et al: Stromal senescence establishes an immunosuppressive microenvironment that drives tumorigenesis. Nat Commun 2016, 7:11762.

40. Gan L, Yang Y, Li Q, Feng Y, Liu T, Guo W: Epigenetic regulation of cancer progression by EZH2: from biological insights to therapeutic potential. Biomark Res 2018, 6:10.

41. Liu YH, Tsai YS, Lin SC, Liao NS, Jan MS, Liang CT, Hsu SW, Chen WC, Sung JM, Maeda N et al: Quantitative PPARy expression affects the balance between tolerance and immunity. Sci Rep 2016, 6:26646.

42. Faucheux L, Grandclaudon M, Perrot-Dockès M, Sirven P, Berger F, Hamy AS, Fourchotte V, VincentSalomon A, Mechta-Grigoriou F, Reyal F et al: A multivariate Th17 metagene for prognostic stratification in T cell non-inflamed triple negative breast cancer. Oncoimmunology 2019, 8(9):e1624130.

\section{Tables}




\begin{tabular}{|c|c|c|c|}
\hline $\begin{array}{l}\text { Immune cell type } \\
\text { in the TME }\end{array}$ & $\begin{array}{l}\text { Immune score-high/stromal } \\
\text { score-low TNBC }(\mathrm{n}=10)\end{array}$ & $\begin{array}{l}\text { Immune score-low/stromal } \\
\text { score-high TNBC }(\mathrm{n}=10)\end{array}$ & $\begin{array}{l}P \text { v- } \\
\text { value* }\end{array}$ \\
\hline naive B cells & $0.0363 \pm 0.0532$ & $0.0258 \pm 0.0263$ & 0.9288 \\
\hline memory B cells & $0.0168 \pm 0.0121$ & $0.0061 \pm 0.0153$ & 0.0304 \\
\hline plasma cells & $0.0162 \pm 0.0376$ & $0.0148 \pm 0.0397$ & 0.7599 \\
\hline CD8+ T cells & $0.1403 \pm 0.0934$ & $0.0531 \pm 0.0616$ & 0.0143 \\
\hline $\begin{array}{l}\text { naive CD4+ T } \\
\text { cells }\end{array}$ & 0 & $\overline{0}$ & \\
\hline $\begin{array}{l}\text { resting CD4+ } \\
\text { memory T cells }\end{array}$ & $0.0956 \pm 0.0557$ & $0.1604 \pm 0.1036$ & 0.2176 \\
\hline $\begin{array}{l}\text { activated CD4+ } \\
\text { memory T cells }\end{array}$ & $0.0441 \pm 0.0316$ & $0.0176 \pm 0.0159$ & 0.0056 \\
\hline $\begin{array}{l}\text { follicular helper T } \\
\text { cells }\end{array}$ & $0.0700 \pm 0.0235$ & $0.0379 \pm 0.0305$ & 0.0044 \\
\hline regulatory T cells & $0.0517 \pm 0.0409$ & $0.0271 \pm 0.0219$ & 0.8767 \\
\hline $\begin{array}{l}\text { gamma delta T } \\
\text { cells }\end{array}$ & $0.0421 \pm 0.0361$ & $0.0259 \pm 0.0268$ & 0.2454 \\
\hline resting NK cells & 0 & $0.0199 \pm 0.0395$ & 0.0108 \\
\hline $\begin{array}{l}\text { activated NK } \\
\text { cells }\end{array}$ & $0.0360 \pm 0.0257$ & $0.0178 \pm 0.0256$ & 0.0511 \\
\hline Monocytes & $0.0009 \pm 0.0030$ & $0.0164 \pm 0.0428$ & 0.2105 \\
\hline M0 macrophages & $0.1196 \pm 0.0540$ & $0.1979 \pm 0.1377$ & 0.2799 \\
\hline M1 macrophages & $0.1497 \pm 0.0789$ & $0.1357 \pm 0.0670$ & 0.8534 \\
\hline M2 macrophages & $0.1043 \pm 0.0628$ & $0.1534 \pm 0.0939$ & 0.1431 \\
\hline $\begin{array}{l}\text { resting dendritic } \\
\text { cells }\end{array}$ & $0.0395 \pm 0.0663$ & $0.0211 \pm 0.0178$ & 0.5889 \\
\hline $\begin{array}{l}\text { activated } \\
\text { dendritic cells }\end{array}$ & $0.0001 \pm 0.0004$ & $0.0008 \pm 0.0026$ & 0.5 \\
\hline resting mast cells & $0.0344 \pm 0.0214$ & $0.0681 \pm 0.0466$ & 0.123 \\
\hline $\begin{array}{l}\text { activated mast } \\
\text { cells }\end{array}$ & 0 & 0 & - \\
\hline eosinophils & $0.0008 \pm 0.0028$ & 0 & $\begin{array}{r}>> \\
0.9999 \\
\end{array}$ \\
\hline neutrophils & $0.0013 \pm 0.0032$ & $0.0002 \pm 0.0006$ & 0.582 \\
\hline
\end{tabular}

(\%) Mean \pm SD; *using Mann-Whitney U test.

CYBERSORTx: https://cibersortx.stanford.edu/index.php 


\begin{tabular}{|c|c|c|}
\hline $\begin{array}{l}\text { Transcription } \\
\text { Factor (TF) }\end{array}$ & Investigated Relationship & PMID \\
\hline PPARG & $\begin{array}{l}\text { PPARy-induced SOCS3 expression prevents IL-17-mediated } \\
\text { cancer growth. }\end{array}$ & 23619236 \\
\hline $\begin{array}{l}\text { CEBPA } \\
(=\mathrm{C} / \mathrm{EBP} \alpha)\end{array}$ & $\begin{array}{l}\text { Transcription factor that coordinates proliferation arrest and the } \\
\text { differentiation of myeloid progenitors, adipocytes, hepatocytes, } \\
\text { and cells of the lung and the placenta. } \\
\text { IL-1 } 7 \text { suppresses expression of several pro-adipogenic TFs, } \\
\text { including PPARy and CEBPA. }\end{array}$ & $\begin{array}{l}23332504 \\
11242107\end{array}$ \\
\hline MEOX1 & $\begin{array}{l}\text { TGF- } \beta 1 \text { transcriptionally regulates MEOX1 expression via } \\
\text { Smad } 2 / 3 \text { in adult human dermal fibroblasts, thus promoting cell } \\
\text { migration. }\end{array}$ & 32241049 \\
\hline KLF15 & $\begin{array}{l}\text { Specifically, IL-17 suppresses KLF15, a pro-adipogenic TF, and } \\
\text { enhances expression of KLF2 and KLF3, which are anti- } \\
\text { adipogenic. Thus, IL-17 suppresses adipogenesis at least in part } \\
\text { through the combined effects of TFs that regulate adipocyte } \\
\text { differentiation. }\end{array}$ & 23332504 \\
\hline CD36 & $\begin{array}{l}\text { In the presence of palmitic acid (PA), IL-17a could directly } \\
\text { increase the cellular uptake of PA, leading to the proliferation of } \\
\text { ovarian cancer cells via the IL-17a/IL-17RA/p-STAT3/FABP4 axis } \\
\text { rather than via CD36. }\end{array}$ & 31802182 \\
\hline ZNF750 & $\begin{array}{l}\text { ZNF750 is the p63 target gene. The levels of inflammatory } \\
\text { cytokines (IL17d and Tnfsf15) were significantly reduced by } \\
\text { Rbm38 deficiency in senescence-resistant Rbm38-/-;TAp63+/- } \\
\text { mouse livers and MEFs. Rbm38 and p63 function as intergenic } \\
\text { suppressors in aging and tumorigenesis. }\end{array}$ & $\begin{array}{l}29520104 \\
24441097\end{array}$ \\
\hline EZH2 & $\begin{array}{l}\text { EZH2 positively regulate the expression of IL-17a and IL-17f. } \\
\text { The inducible binding of EZH2 at the IL-17a promoter was } \\
\text { dependent on signaling pathways downstream of the TCR. } \\
\text { IL-17f bears } 50 \% \text { homology to IL-17a and has recently been } \\
\text { suggested to play a role in inflammation. }\end{array}$ & $\begin{array}{l}21674483 \\
31850514\end{array}$ \\
\hline $\begin{array}{l}\text { HNF4A (= } \\
\text { farnesoid X } \\
\text { receptor, } \\
\text { FXR) }\end{array}$ & $\begin{array}{l}\text { In addition to the classical Jak-Stat antiviral signaling pathway, } \\
\text { IFN- } 1 \text { inhibits hepatitis } C \text { virus replication through the } \\
\text { suppression of miRNA-122 transcription via an inflammatory Stat } \\
\text { 3-HNF4A feedback loop. Inflammatory feedback circuits } \\
\text { activated by IFNs during chronic inflammation expose non- } \\
\text { responders to the risk of hepatocellular carcinoma. }\end{array}$ & 26657215 \\
\hline $\begin{array}{l}\text { NR0B2 (= } \\
\text { SHP) }\end{array}$ & $\begin{array}{l}\text { This TF is a tumor suppressor. Both FXR (-/-) and NR0B2(-/-) } \\
\text { mice develop spontaneous hepatocellular carcinoma. } \\
\text { Upregulated NR0B2 will regulate the IL-6-dependent pathway. }\end{array}$ & $\begin{array}{l}23811326 \\
22349108\end{array}$ \\
\hline
\end{tabular}

\section{Figures}


TCGA-Breast Ca. gene expression data

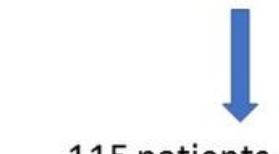

115 patients with TNBC

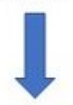

Estimate immune and stromal cells in TME

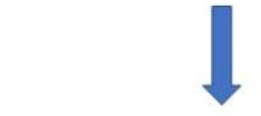

Correlate Stromal/Immune

Scores with prognosis

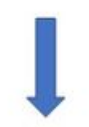

Compare proportion of immune cells by CIBERSORTX
Modeling into two

prognostically distinct groups

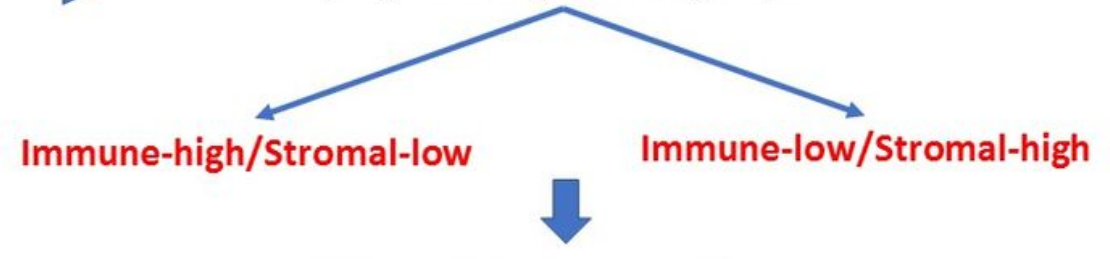

Differentially Expressed Genes
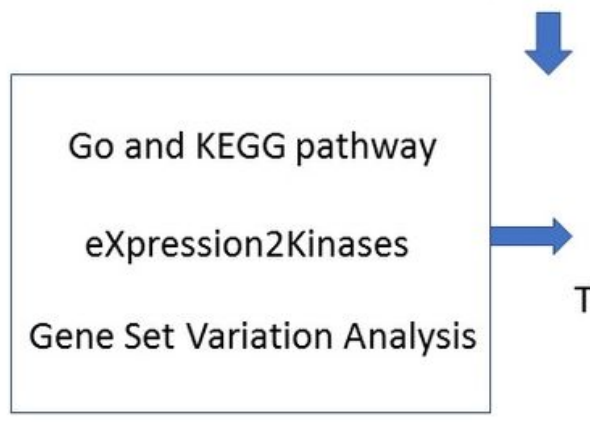

Signatures of differentially expressed genes

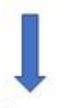

Transcriptional factors Enrichment

Validation in 5,793 patients via cBioPortal

\section{Figure 1}

Study flow diagram. The schematic diagram represents the study design and methodology adopted. 

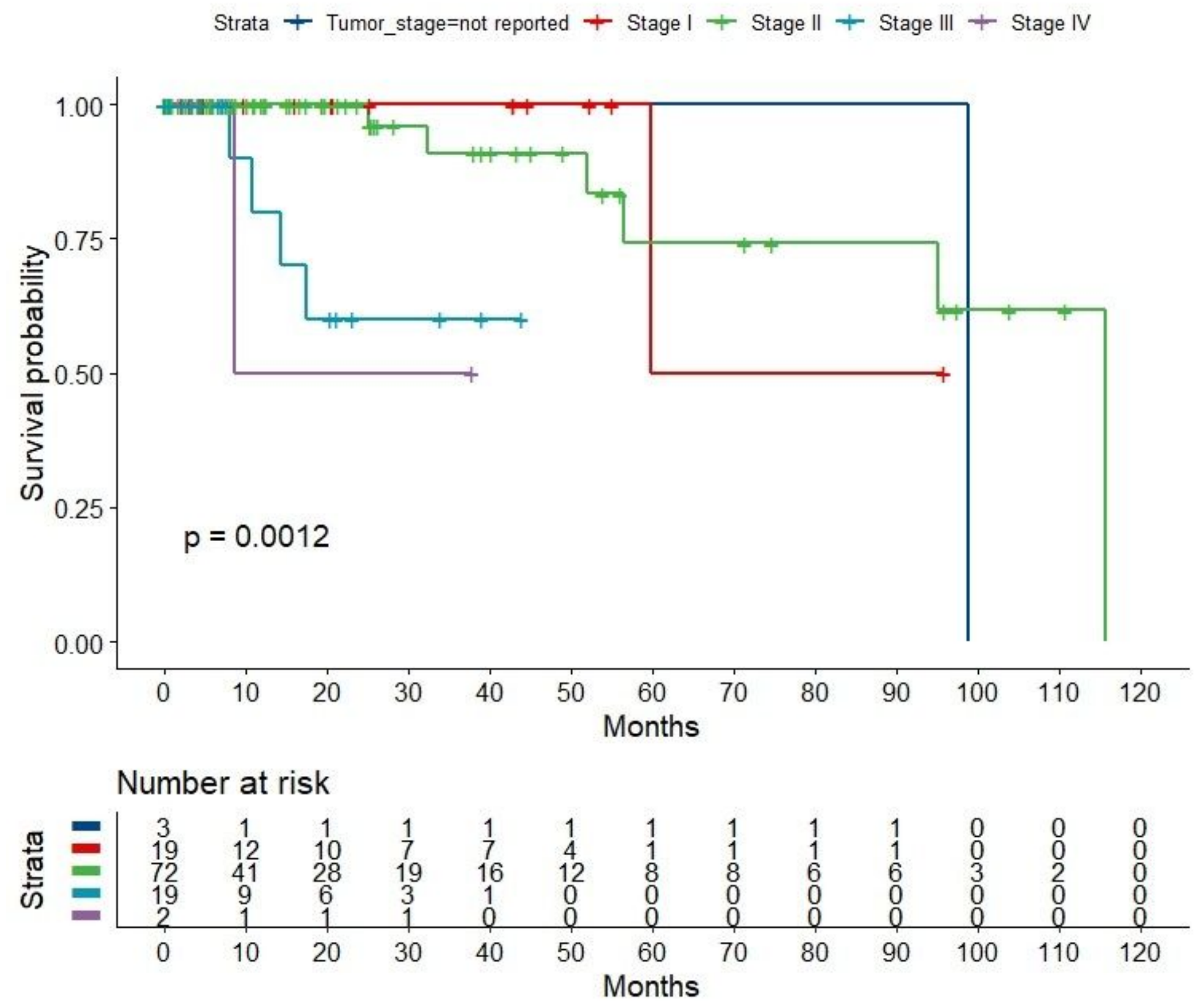

Figure 2

Overall survival of 115 patients with triple-negative breast cancer stratified by cancer stage at diagnosis. Kaplan-Meier curves referring to different overall TNBC stages. The higher stage correlates significantly with poorer survival $(P=0.0012)$. 


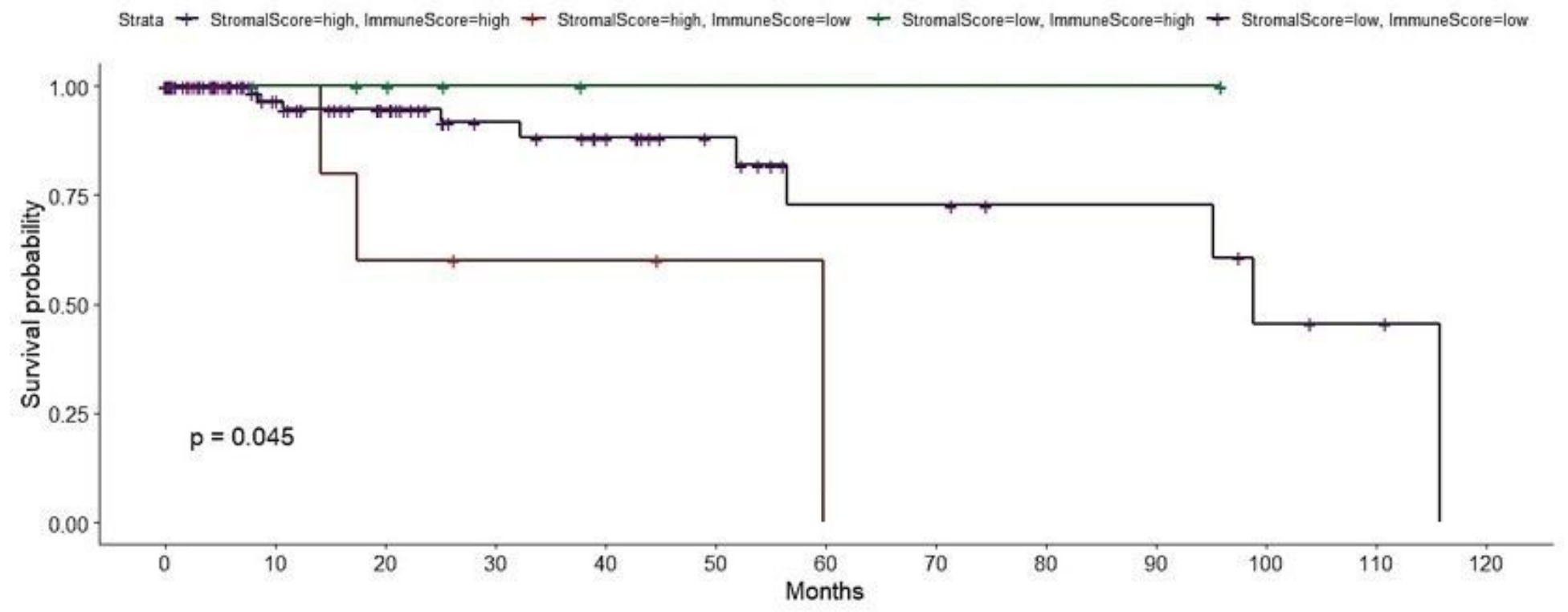

Number at risk

\section{Figure 3}

Overall survival of 115 patients with triple-negative breast cancer stratified by the combination of the Stromal Score and Immune Score. Both scores were inferred by the ESTIMATE gene expression signatures. SS-low/IS-high patients were associated with excellent overall survival, whereas SS-high/ISlow patients showed the worst overall survival. 
A

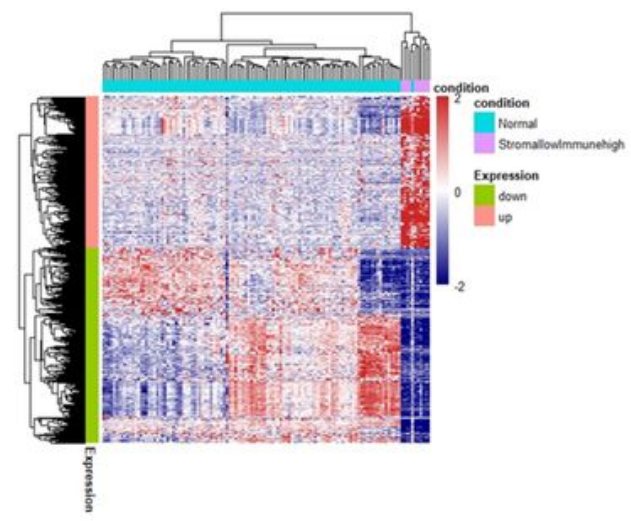

C

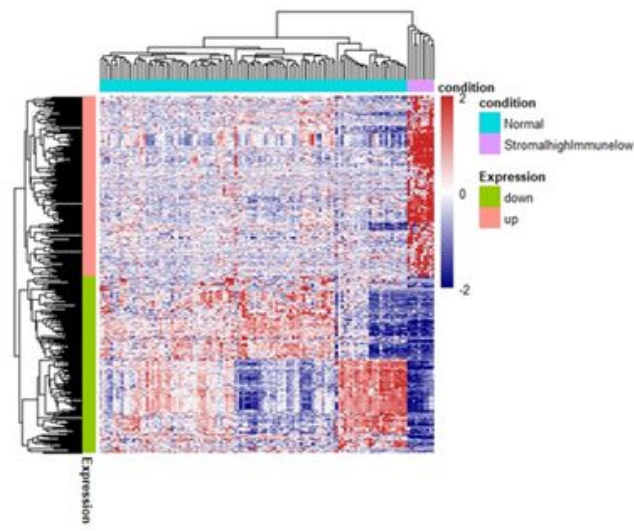

E

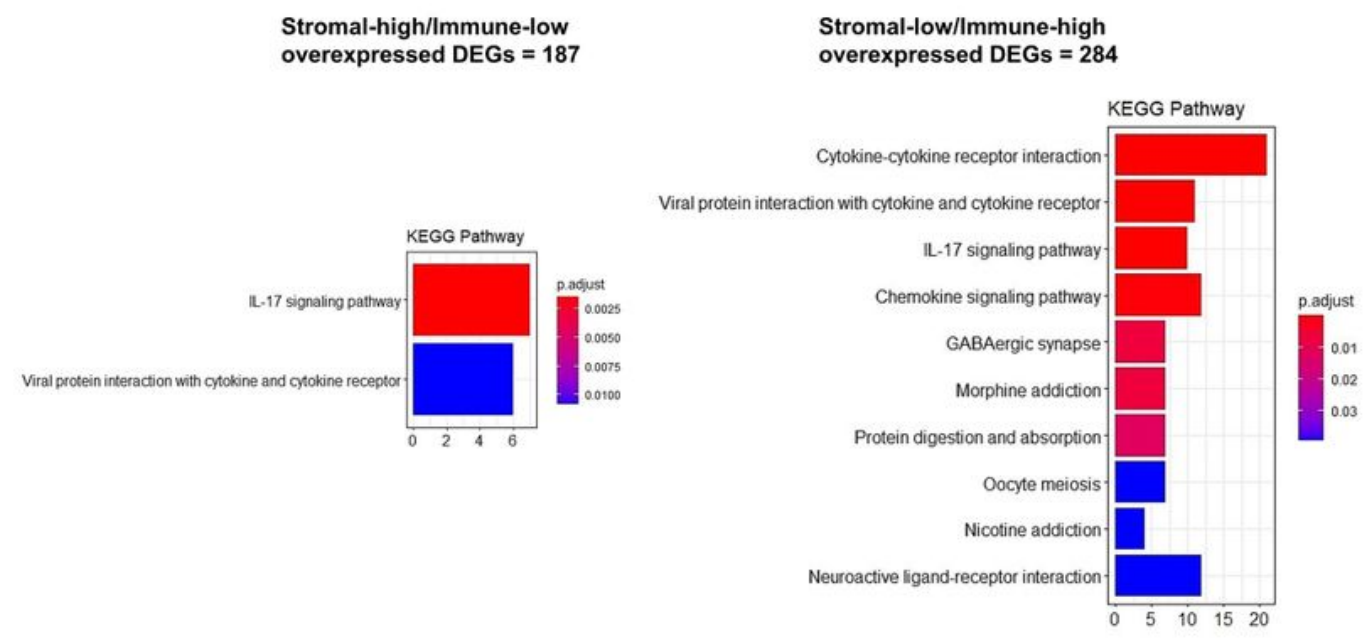

B

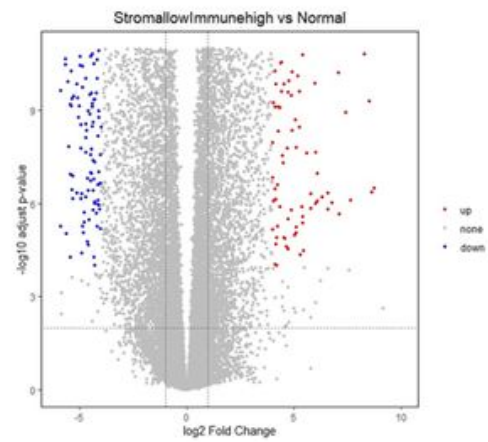

D

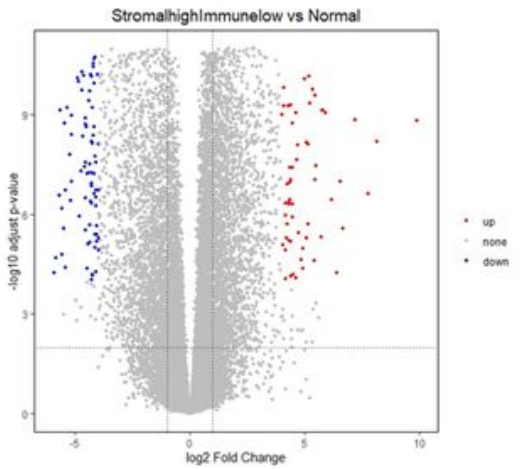




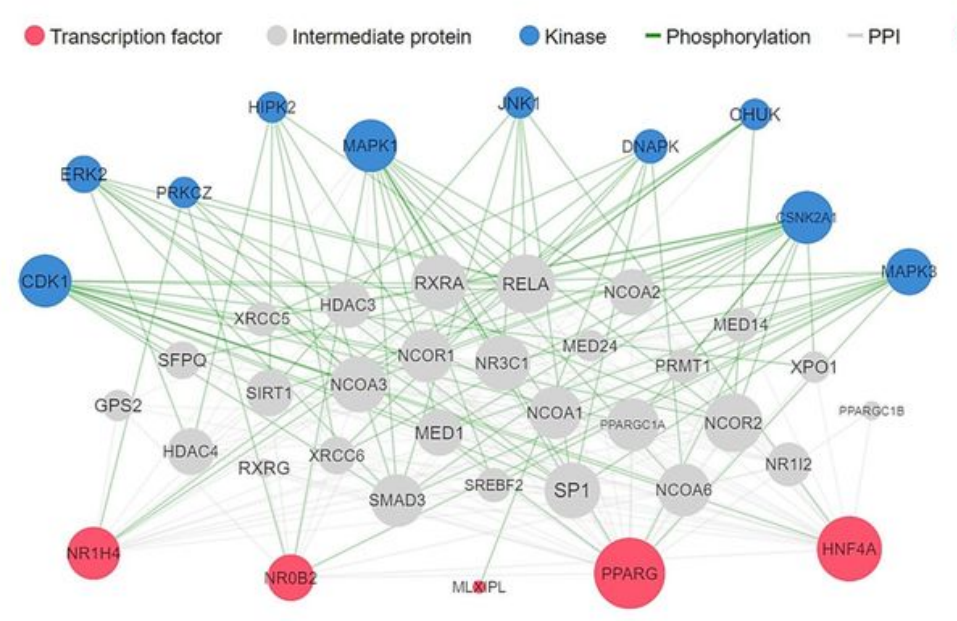

Stromal Score-high/Immune Score-low TNBC

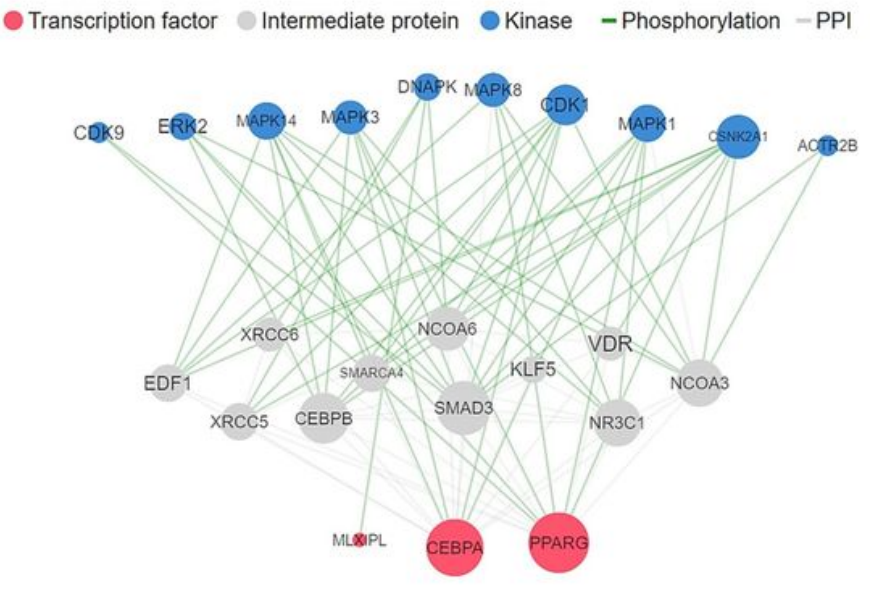

Stromal Score-low/Immune Score-high TNBC

\section{Figure 5}

Transcription factors inferred from the X2K in the context of SS-high/IS-low or SS-low/IS-high TNBC patients.
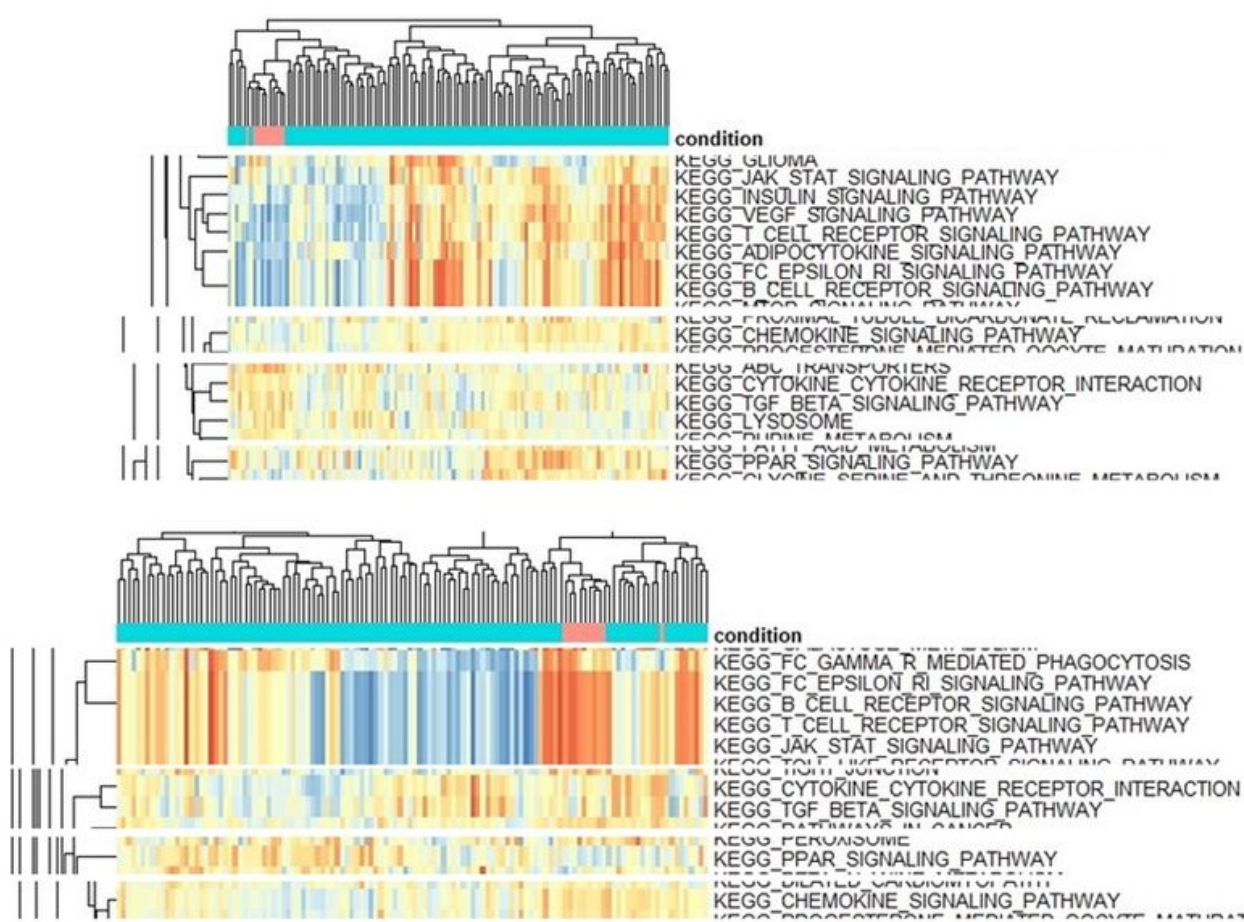

$\| 1 \mid 4_{H}$ condition

KEGG_B_CELL_RECEPTOR_SIGNALING PATHWAY

KEGG_TCELL_RECEPTOR SIGNALING PATHWAY

KEGG JAK STAT SIGNALING PATHWAY

KEGG_CYTOEIINE_CYTOKINE RECEPTOR_INTERACTION

KEGG_TGF BETA-SIGNALING PATHWAY

KEGG_PPAR_SIGNALING PATHWAY

KEGG CHEMOKINE SIGNALING PATHWAY
KEGG $-\overline{F C}$ GAMMA $R$ MËDIATED PHAGOCYTOSIS
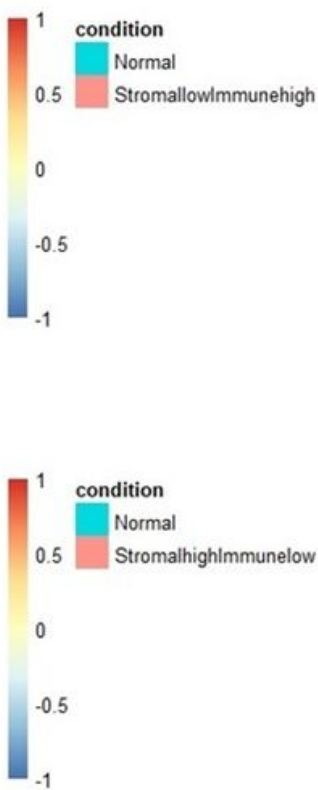

\section{Figure 6}

Gene Set Variation Analysis showing the differential pathway activity. The upper panel reveals the GSVA of the 370 DEGs of the SS-high/IS-low subgroup. The lower panel shows the GSVA of the 651 DEGs of 
the SS-low/IS-high subgroup. Both demonstrate the gene enrichment in the JAK-STAT signaling, T cell receptor signaling, B cell receptor signaling, cytokine-cytokine receptor interaction, TGF- $\beta$ signaling, and PPAR signaling pathways.
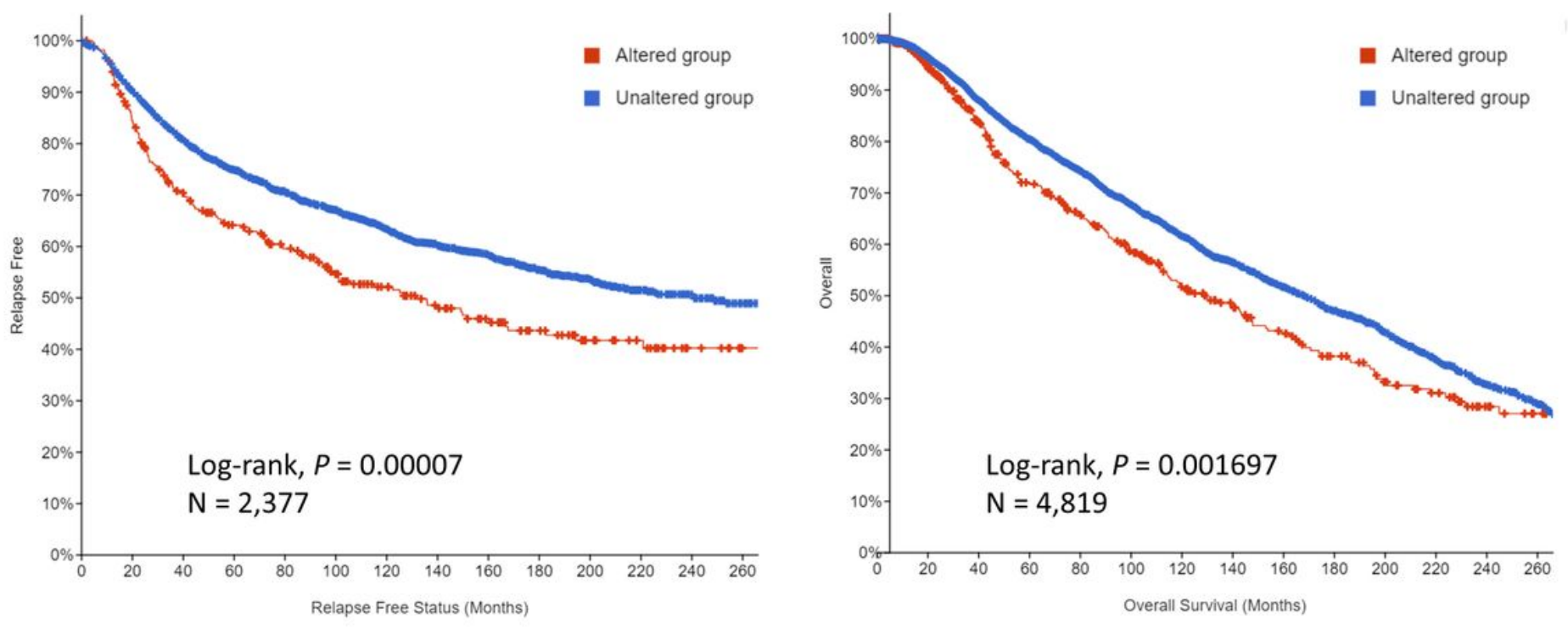

\begin{tabular}{|l|l|l|l|l|l|r|}
\hline Survival Type & $\begin{array}{l}\text { No. } \\
\text { Patients } \\
\text { with Data }\end{array}$ & $\begin{array}{l}\text { No. in } \\
\text { Altered } \\
\text { group }\end{array}$ & $\begin{array}{l}\text { No. in } \\
\text { Unaltered } \\
\text { group }\end{array}$ & $\begin{array}{l}\text { Median } \\
\text { survival in } \\
\text { Altered }\end{array}$ & $\begin{array}{l}\text { Median } \\
\text { survival in } \\
\text { Unaltered }\end{array}$ & $\boldsymbol{p}$-Value \\
\hline Relapse- Free & 2,377 & 282 & 2,095 & $131.71 \mathrm{mo}$. & $240.95 \mathrm{mo}$. & 0.00007 \\
\hline Overall & 4,819 & 465 & 4,354 & $129.6 \mathrm{mo}$. & $168.2 \mathrm{mo}$. & 0.001697 \\
\hline
\end{tabular}

\section{Figure 7}

Validation using the cBioPortal demonstrates the relapse-free survival in 2,377 patients and the overall survival in 4,819 patients with invasive breast carcinoma stratified based on the alteration of the transcription factor genes PPARG, CEBPA, MEOX1, CD36, ZNF750, KLF15, EZH2, HNF4A, and NR0B2.

\section{Supplementary Files}

This is a list of supplementary files associated with this preprint. Click to download.

- SuppTable1.docx

- SupplFig1.ImmuneScoreandStromalScorebystages.tif

- SupplFig2.SurvivalbyStromalScoreloworhighNew.jpg

- SupplFig3.jpg

- SupplFig4.tif

- SupplFig5New.tif

- SupplFig6.pdf

- SupplFig70ncoprintforlL17genesaberration.tif 